\title{
In Vitro Activity Screening of Snake Venom against Multi Drug Resistant Tuberculosis
}

\author{
Sujay Kumar Bhunia1, Mrinmoy Sarkar ${ }^{1}$, Sananda Dey ${ }^{1}$, Arpita Bhakta $^{2}$, Antony Gomes ${ }^{3}$ and Biplab Giri ${ }^{*}$ \\ ${ }^{1}$ Experimental Medicine \& Stem Cell Research Laboratory, Department of Physiology, West Bengal State University, Barasat, Kolkata 700 126, India \\ ${ }^{2}$ Department of Laboratory Medicine, AMRI Hospital, Goriahat Road, Kolkata 700031 , India \\ ${ }^{3}$ Laboratory of Toxicology \& Experimental Pharmacodynamics, Department of Physiology, University of Calcutta, 92 APC Road, Kolkata 700 009, India
}

\begin{abstract}
The re-emergence of multidrug-resistant tuberculosis (MDR-TB) events has brought to light the importance of screening effective novel drugs. In the present study, in vitro activities of different snake (Naja naja, Bungarus fasciatus, Daboia russelli russelli, Naja kaouthia) venoms have been investigated against clinical isolate of MDR-TB strains. All the venoms inhibited the mycobacterial growth for at least a week in common and two of them (Naja naja, Naja kaouthia) showed significantly longer inhibition up to two weeks against the MDR-TB strain with single dose and one repetition of those two venoms exhibited inhibition up to more than 4 weeks.
\end{abstract}

Keywords: MDR-TB; Multidrug resistance; Tuberculosis; Snake venom

\section{Short Communication}

Tuberculosis (TB), caused by Mycobacterium tuberculosis (Mtb), is one of the world's major health problems and still remains most common and deadly infectious disease in developing countries. According to World Health Organization (WHO) report (2013), there were an estimated 8.6 million people developed TB and 1.3 million died from the disease in 2012 globally [1]. It is projected in a previous report that nearly one-third of the world's population is infected and more than 1.5 million people die of TB every year [2]. Among the worldwide incidents of TB, $2-3.7 \%$ are estimated to have multidrug resistant tuberculosis (MDR-TB) with almost 0.5 million MDR-TB cases emerging each year worldwide and between $5 \%$ and $7 \%$ of them becoming extensively drug resistant tuberculosis (XDR-TB) [2-6]. MDR-TB is resistant to isoniazid (INH) and rifampicin (RMP), with or without resistance to any other anti-tuberculosis drug. Treatment for MDR-TB is much less effective than treatment for drug-susceptible TB, since it is extensively lengthy and expensive and has an even poorer success rate of only $48 \%$ worldwide, as it was in $2010[7,8]$. The by and large bacteriostatic second line drugs have a lower efficacy than the first line anti-TB drugs and hence they do consume longer time to treat MDR and XDR-TB. Moreover, considering the association of HIV with MDR-TB in the present scenario, the inconvenience arises when these second-line drugs apart from being expensive, and toxic, these are found difficult to combine with antiretroviral drugs, and also are unavailable in most of the parts of developing countries $[9,10]$. Therefore in the current circumstances, MDR-TB continues to be a formidable public health challenge worldwide and measures has to be taken immediately because current range of vaccines and chemotherapeutic treatments are limited in their efficacy and fail to prevent the spread of the disease. Therefore, there is an urgent need for new, inexpensive anti-MDR/ XDR-TB drugs which are more effective and with fewer side effects.

Medicinal plants and animal toxins offer a great hope to fulfill these needs and have been used as a natural source for curing diseases for many centuries. Putting aside the relatively lethal role of venoms and toxins in global morbidity and mortality, they can be attributed to be useful as potential therapeutic probe for illuminating multifaceted biological processes. In line with this, it is reported that snake venom are also loaded with biologically active components [11-13]. Therefore, to meet the pressing need to develop high efficacy drugs against both drug-sensitive and drug-resistant Mtb strains from some natural source we are presenting our report showing snake venom screening for activity against clinically isolated MDR-TB.

The culture media used for Mtb growth was Lowenstein Jensen (L-J) medium (Sigma-Aldrich ${ }^{\circ}$, United States) and Middle brook 7H9 broth (Becton-Dickinson; New Jersey, United States) were used for the sensitivity test. Both were prepared according to the manufacturer's instruction. The reference strain Mycobacterium tuberculosis subsp. Tuberculosis, TMC 102 [H37Rv] $\left(\right.$ ATCC $^{\oplus} 27294^{\text {tix }}$ ) was obtained from American Type Culture Collection (ATCC, USA) and the clinical isolate drug resistant strain of $\mathrm{M}$. tuberculosis was obtained from the Department of Laboratory Medicine (Microbiology Division) of Advanced Medical Research Institute (AMRI) hospital, Kolkata. The bacteria were incubated at $37^{\circ} \mathrm{C}$ and grown in $7 \mathrm{H} 9$ Middlebrook TB medium containing 14C-labelled palmitic acid as a source of carbon.

\section{LJ Media Tubes}

LJ slant was inoculated with the $0.1 \mathrm{ml}$ of the processed sample and were incubated at $37^{\circ} \mathrm{C}$ for a maximum of 8 weeks. They were checked twice weekly for first two weeks and then once every week for maximum period of 8 weeks. Bacterial contamination was examined by performing Gram staining from the suspected colonies.

\section{Preparation of the Isolate from Solid Media}

The drug resistant clinical isolate of $\mathrm{Mtb}$ was obtained from Department of Laboratory Medicine (Microbiology Division) of (AMRI) hospital, Kolkata. $4 \mathrm{ml}$ of BBL Middlebrook 7H9 Broth was added to a $16.5 \times 128 \mathrm{~mm}$ capped sterile tube, containing 8-10 glass beads. Many colonies, not more than 14 days old, were then scrapped from the MDR growth slant (Löwenstein-Jensen media) with a sterile

${ }^{*}$ Corresponding author: Biplab Giri, Experimental Medicine \& Stem Cell Research Laboratory, Department of Physiology, West Bengal State University, Barasat, Kolkata 700 126, India, Tel/Fax: 91 33-2524 1977; E-mail: bgiri.emscrl@gmail.com

Received November 15, 2015; Accepted December 08, 2015; Published December 15, 2015

Citation: Bhunia SK, Sarkar M, Dey S, Bhakta A, Gomes A, et al. (2015) In Vitro Activity Screening of Snake Venom against Multi Drug Resistant Tuberculosis. J Trop Dis 4: 192. doi:10.4172/2329-891X.1000192

Copyright: (c) 2015 Bhunia SK, et al. This is an open-access article distributed under the terms of the Creative Commons Attribution License, which permits unrestricted use, distribution, and reproduction in any medium, provided the original author and source are credited. 
loop, and were suspended in the Middlebrook 7H9Broth. The tube was vortexed for 2-3 minutes to suspend the larger clumps. The suspension thus prepared exceeded a $1.0 \mathrm{McF}$ arland standard in turbidity. It was then left undisturbed for 20 minutes at room temperature to get the supernatant. The supernatant fluid was transferred to another $16.5 \mathrm{x}$ $128 \mathrm{~mm}$ capped sterile tube and left to precipitate for another $15 \mathrm{~min}$. The supernatant fluid was finally transferred (it should be smooth, free of any clumps) to a third $16.5 \times 128 \mathrm{~mm}$ sterile tube. The suspension was adjusted to a 0.5 McFarland by means of a visual comparison to a 0.5 McFarland turbidity standard. The adjusted suspension was diluted 5 times with sterile saline before the sensitivity test.

The snake venoms were collected from the Laboratory of Toxicology \& Experimental Pharmacodynamics (Dept. of Physiology, University of Calcutta). Indian cobra (Naja naja) venom (NN), banded krait (Bungarus fasciatus) venom (BF), Indian viper (Daboia russelli russelli) venom (DRR), and Indian monocellate cobra (Naja kaouthia) venom (NK) were used in this study. A primary stock was prepared using lyophilized venom and diluted as $1 \mathrm{mg} / \mathrm{ml}$ solution with deionized sterile water. From the stock solution, various concentrations $(5 \mu \mathrm{g} / \mathrm{ml}$, $10 \mu \mathrm{g} / \mathrm{ml}$ and $20 \mu \mathrm{g} / \mathrm{ml}$ ) of the venoms were used for the susceptibility testing.

\section{MGIT Tube}

The BBL MGIT tube (Becton Dickinson, USA) containing $7 \mathrm{ml}$ modified middle brook 7H9 broth was used, to which an enrichment supplement Oleic Albumin Dextrose Catalase (OADC; BD, USA) was added. After inoculation, the tubes were incubated at $37^{\circ} \mathrm{C}$. Readings were taken daily for 15 days for culture positivity using the BBL Micro MGIT system. All the culture positive tube was further confirmed by Ziehl-Neelsen (ZN) staining and a sub-culturing on blood agar plate and a LJ slant. The time to detection (TTD) of Mycobacteria was based on the date of the earliest instrumental indication of positivity. The BACTEC MGIT960 susceptibility method is a $4-13$ day qualitative test (according to manufacturer's instruction). The test is based on growth of the Mtb isolate in a drug-containing tube compared to a drug-free

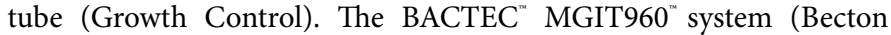
Dickinson, USA) automatically interprets these results and reports a susceptible or resistant result.

The Micro MGIT system detected mycobacteria significantly earlier than LJ medium. For smear positive specimens, the mean turn-around time was 8 days by Micro MGIT whereas on LJ medium, it was 36 days [14]. Thereafter, using Middle brook 7H9 broth, for detection of anti MDR-TB activity of the various snake venoms (NN, BF, DRR, NK), it was observed that all of the used venoms showed any certain degree of anti-MDR-TB activity with a single dose of treatment. Using identical microenvironment and treatment schedule all the substances inhibited the growth of MDR-TB for at least 8 days in common and two of the agents showed highest inhibition, for 14 days, at a concentration of 20 $\mu \mathrm{g} / \mathrm{ml}$ (Figure 1a). We therefore, went on to investigate more regarding the effectiveness of the second similar dose of the venoms which showed inhibition of the pathogen for the longest period, around 2 weeks. The repetition of the dosage at $8^{\text {th }}$ day resulted in inhibition of the MDR-TB strain for around 4 weeks (Figure $1 \mathrm{~b}$ ) for NN and NK venoms, whereas the BF and DRR did not cross more than 2 weeks of effectiveness.

The data was presented here (Figure 1a and $1 \mathrm{~b}$ ) based on the data obtained from the instrument as interpreted according to the manufacturer's instructions as $1-12=$ negative for culture, $13=$ intermediate and $14-20=$ positive for culture. Tuberculosis (TB) is a serious public health problem with medical, sociological and economic consequences. Drug susceptibility testing for TB patients is one of the most effective tools of control and management of MDR-TB. Drug susceptibility testing for all TB cases to provide optimal treatment, establishing advanced diagnostic facilities for rapid detection of MDR$\mathrm{TB}$, continuous monitoring of drug resistance and control of drug resistant $\mathrm{TB}$ at border entry points with high-TB burden countries are recommended for prevention and control of drug-resistant TB. Natural products such as plant-derived molecules have long been valuable sources for novel medicine and previously reported as antimycobacterial agents [15,16]. Xie et al. (2003) has showed in vitro activities of small peptides, derived from Naja atra, to have useful activity against MDR-TB [17].

In this study, we found $\mathrm{NN}, \mathrm{BF}, \mathrm{DRR}$ and $\mathrm{NK}$ snake venoms demonstrated a dose dependent inhibition of the MDR-TB isolates in Middlebrook 7H9 media. Snake venom is rich sources of small peptides with specific functions $[11,12,13,17]$. It was observed that $\mathrm{NN}$ and $\mathrm{NK}$ showed anti-mycobacterial activity up to 14 days of observation in a single dose of treatment as compared to control. Interestingly, with the repetition of similar doses of NN, BF, DRR and NK venoms on the $8^{\text {th }}$ day of effectiveness, inhibition by NN and NK venoms could be observed for 28 and 31 days respectively. After that the pathogens rejuvenated themselves, may be due to the decomposition of the venoms in the prolonged exposure at $37^{\circ} \mathrm{C}$. In case of DRR venom, the $10 \mu \mathrm{g} / \mathrm{ml}$ dose showed an apparent higher effectivity, though insignificant, when compared the mean of days of effectiveness with that of the $5 \mu \mathrm{g} / \mathrm{ml}$ dose but the $20 \mu \mathrm{g} / \mathrm{ml}$ did not show any further change in the effectiveness of the venom in inhibition of the pathogen. While, comparing the three different doses of BF venom, a significant change was only observed when we compared the mean of days of effectiveness in the cases where $5 \mu \mathrm{g} / \mathrm{ml}$ and $20 \mu \mathrm{g} / \mathrm{ml}$ doses had been used. But in contrast to the lower effectiveness of the aforesaid venoms, significantly longer duration of effectively was observed in the venoms from NN and NK. The $5 \mu \mathrm{g} / \mathrm{ml}$ dose of NK venom though did not show significant change in longevity of its inhibitory effect towards the growth of pathogen when compared with the same dose of other venoms, the similar dose of NN venoms had a significantly longer effectively compared to the DRR and BF venoms. In line with this, the higher doses of $\mathrm{NN}$ and NK venoms showed a significantly $\left({ }^{* * *} p<0.001\right)$ longer duration of effectively as compared to the DRR and BF venoms. Since, the pathogen started growing again after 14 days of single treatment; we became curious whether repetition of doses could be more effective. Therefore, we introduced a second similar dose at $8^{\text {th }}$ day of $1^{\text {st }}$ treatment for all the samples only to find a more or less similar consequence of effectiveness of the venoms.

In our previous review regarding the life-hunting disease and ways of treatment and management we illustrated various combat processes [18]. In agreement with that, the conclusion of this study would justify the need for further investigations on the correlation between structural features and anti-tuberculosis activity as some bioactive molecules which have proved useful as model compounds or templates can be employed in the synthesis or semi-synthesis of new drugs. And the results of our study, showing all of the snake venom crude substances having anti MDR-TB activities, may be explored for development of new anti-TB drugs. In our earlier study, we have established cytotoxic effects of Naja kaouthia venom derived peptide (NK-CT1) and the amino acid sequence have been explored which has sequence homology with other cytotoxins (cytotoxin-3, CTX-6, cytotoxin-2, CTX-A3) isolated from different snake venoms [12]. Cytotoxins have the ability to damage a wide variety of cells including the cancerous types. Therefore, further in vitro evaluation of anti-MDR-TB factor(s) from Naja kaouthia and Naja naja snake venoms including NK-CT1 would be of our prime interest. 
Citation: Bhunia SK, Sarkar M, Dey S, Bhakta A, Gomes A, et al. (2015) In Vitro Activity Screening of Snake Venom against Multi Drug Resistant Tuberculosis. J Trop Dis 4: 192. doi:10.4172/2329-891X.1000192

(a)
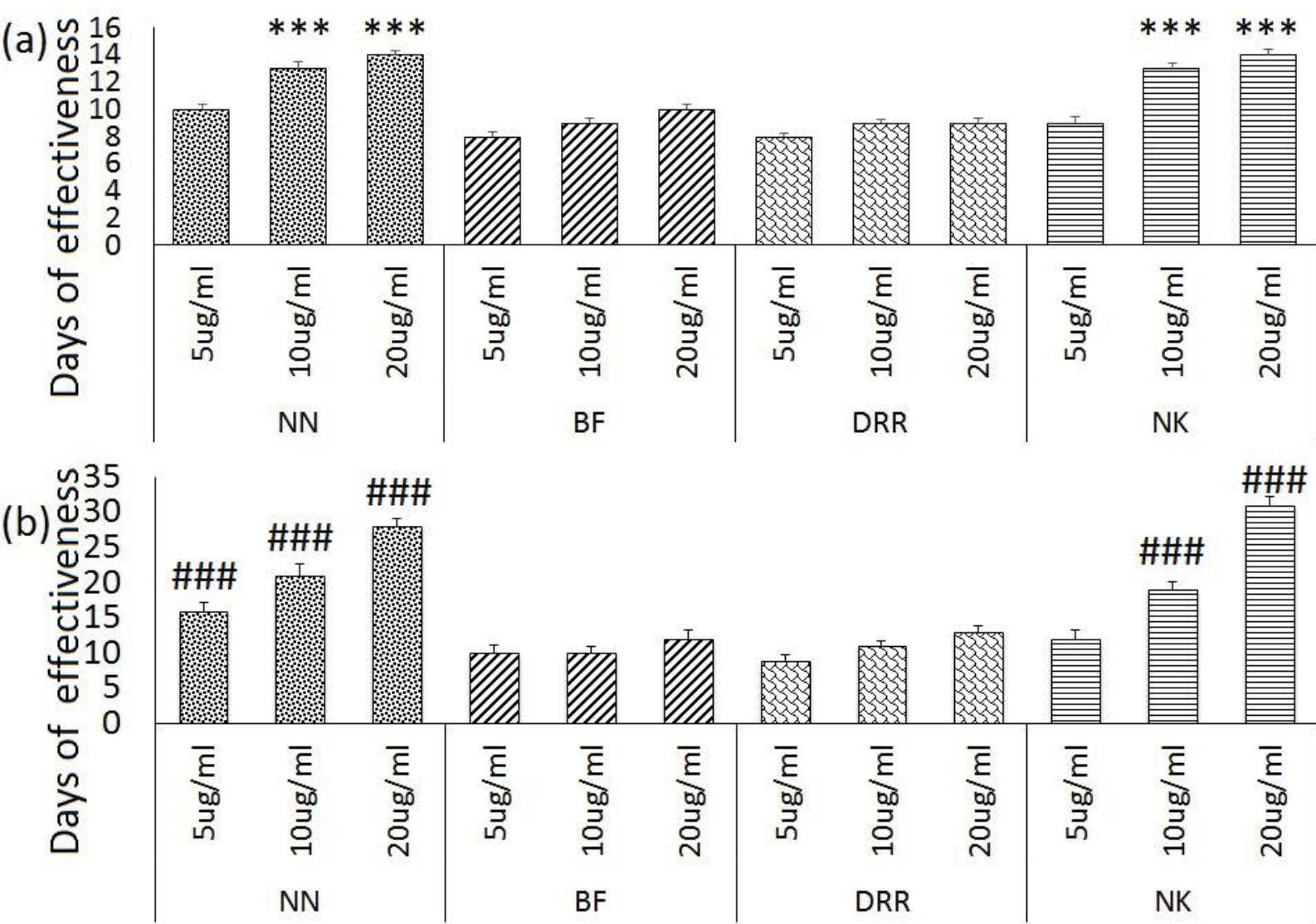

Figure 1: Anti-mycobacterial activity of different snake venoms against multidrug-resistant tuberculosis strain of bacteria. 1/100 dilution of control mycobacterium culture tube was compared each day with microDigit data and represented as effectiveness in terms of number of days effective with single dose of venom treatment (a) and with repetition of treatment $(\mathrm{b})$ at $8^{\text {th }}$ day of $1^{\text {st }}$ treatment respectively. Data represented here as mean $\pm S D$. All the data were significant when compared with their respective control values as per the instruments' instruction manual.

(a) ${ }^{* * *} p<0.001$ [days of effectiveness of NN $(10 \& 20 \mu \mathrm{g} / \mathrm{ml})$ and NK $(10 \& 20 \mu \mathrm{g} / \mathrm{ml}) v s$. days of effectiveness of respective doses of DRR and BF]

(b) ${ }^{\#} p<0.001$ [days of effectiveness of NN $(5,10 \& 20 \mu \mathrm{g} / \mathrm{ml})$ and NK (10 \& $\left.20 \mu \mathrm{g} / \mathrm{ml}\right) v s$. days of effectiveness of respective doses of DRR and BF]

\section{Acknowledgement}

Authors gratefully acknowledge AMRI Hospital for providing us their machine for the present study and we also thankful to the West Bengal State University for providing us necessary infrastructure and facilities. This paper is partially supported by the grants of Dr. Biplab Giri [SERB (DST), (SR/FT-132/2010), Govt. of India].

\section{References}

1. Dinh TH, Delaney KP, Goga A, Jackson D, Lombard 5, et al. (2015) Impact of Maternal HIV Seroconversion during Pregnancy on Early Mother to Child Transmission of HIV (MTCT) Measured at 4-8 Weeks Postpartum in South Africa 2011-2012: A National Population-Based Evaluation. PLoS One 10: e0125525.

2. World Health Organization (2004) Anti-tuberculosis drug resistance in the world. TheWHO/IUATLD project on anti-tuberculosis drug resistance surveillance, Geneva.

3. Wehrli W, Staehelin M (1971) Actions of the rifamycins. See comment in PubMed Commons below Bacteriol Rev 35: 290-309.

4. WHO (2008) Anti-Tuberculosis Drug Resistance in the World.
5. World Health Organization (2010) Multidrug and Extensively Drug-Resistant TB (M/XDR-TB). 2010 Global Report on Surveillance and Response.

6. World Health Organisation (2013) Global tuberculosis report.

7. Ahuja SD, Ashkin D, Avendano M, Banerjee R, Bauer M, et al. (2012) Multidrug resistant pulmonary tuberculosis treatment regimens and patient outcomes: an individual patient data meta-analysis of 9,153 patients. PLoS Med 9: e1001300.

8. Dean GL, Edwards SG, Ives NJ, Matthews G, Fox EF, et al. (2002) Treatment of tuberculosis in HIV-infected persons in the era of highly active antiretroviral therapy. AIDS 16: 75-83.

9. Carroll MW, Lee M, Cai Y, Hallahan CW, Shaw PA, et al. (2012) Frequency of adverse reactions to first- and second-line anti-tuberculosis chemotherapy in a Korean cohort. See comment in PubMed Commons below Int J Tuberc Lung Dis 16: 961-966.

10. Gomes A, Bhattacharjee P, Mishra R, Biswas AK, Dasgupta SC, et al. (2010) Anticancer potential of animal venoms and toxins. Ind J Exp Biol 48: 93-103.

11. Debnath A, Saha A, Gomes A, Biswas S, Chakrabarti P, et al. (2010) A letha cardiotoxic-cytotoxic protein from the Indian monocellate cobra (Naja kaouthia) venom. Toxicon 56: 569-579. 
Citation: Bhunia SK, Sarkar M, Dey S, Bhakta A, Gomes A, et al. (2015) In Vitro Activity Screening of Snake Venom against Multi Drug Resistant Tuberculosis. J Trop Dis 4: 192. doi:10.4172/2329-891X.1000192

12. Gomes A, Choudhury SR, Saha A, Mishra R, Giri B, et al. (2007) A heat stable protein toxin (drCT-I) from the Indian Viper (Daboia russelli russelli) venom having antiproliferative, cytotoxic and apoptotic activities. Toxicon 49: 46-56.

13. Akintola AO, Kehinde AO, Adebiyi OE, Ademowo OG (2013) Anti-tuberculos activities of the crude methanolic extract and purified fractions of the bulb of Crinum jagus. Niger J Physiol Sci 28: 135-140.

14. Lau N, Meyer JM (1999) In vitro inhibition of drug-resistant and drug sensitive strains of Mycobacterium tuberculosis by ethnobotanically selected South Africa plants. J Ethnopharmacol 66: 347-354.

15. Zhou GC, Komatsu K, Yaqmaji S, Namba T (1994) Pharmacognostical studies on the traditional drugs used by Chinese minority race, called ' $\mathrm{Yl}$ ' or 'A-ji-bamo'derived from Dipsacus plants. Nat Med 48: 131- 140

16. Xie JP, Yue J, Xiong YL, Wang WY, Yu SQ, et al. (2003) In vitro activities of small peptides from snake venom against clinical isolates of drug-resistant Mycobacterium tuberculosis. Int J Antimicro Agents 22: 172-174.

17. Bhunia SK, Sarkar M, Banerjee A, Giri B (2015) An Update on Pathogenesis and Management of Tuberculosis with Special Reference to Drug Resistance. Asian Pacific Journal of Tropical Disease 5: 673-686.

18. Feofanov AV, Sharonov GV, Astapova MV, Rodionov DI, Utkin YN, et al. (2005) Cancer cell injury by cytotoxins from cobra venom is mediated through lysosomal damage. Biochem J 390: 11-18. 